\title{
Preliminary study for the stimulation effect of plant-based meals on pure culture Lactobacillus plantarum growth and acidification in milk fermentation
}

\author{
Feng Hang, ${ }^{1,2,3 *} \odot$ Yuanzhi Jiang, ${ }^{1,2,3} \odot$ Liwen Yan, ${ }^{3} \odot$ Qing Hong, ${ }^{2} \odot$ Wenwei Lu,,${ }^{1,2,4} \oplus$ Jianxin Zhao, ${ }^{1,2,4} \oplus$ \\ Hao Zhang, ${ }^{1,2,3,4}$ (1) and Wei Chen ${ }^{1,2,4,5}$ (1) \\ ${ }^{1}$ State Key Laboratory of Food Science and Technology, School of Food Science and Technology, Jiangnan University, Wuxi, Jiangsu 214122 , \\ China \\ ${ }^{2}$ School of Food Science and Technology, Jiangnan University, Wuxi, Jiangsu 214122, China \\ ${ }^{3}$ Food Biotechnology Institute of Jiangnan University, Yangzhou, Jiangsu 225004, China \\ ${ }^{4}$ National Engineering Research Center for Functional Food, Jiangnan University, Wuxi, Jiangsu 214122, China \\ ${ }^{5}$ Beijing Innovation Centre of Food Nutrition and Human Health, Beijing Technology and Business University, Beijing 100048, China
}

\section{ABSTRACT}

Fermented dairy products have been recognized as the best carriers for the administration of probiotics. Because one of the potential probiotic strains, Lactobacillus plantarum, has poor proteolytic ability and weak acidifying capacity in milk fermentation, the aim of this study was to preliminarily investigate the stimulation effect of plant-based meals on L. plantarum CCFM8661 growth in milk, and subsequently develop a yogurt or yogurt drinks containing probiotic strain L. plantarum CCFM8661. Milk supplemented with different concentrations ( 5 to $10 \%$, wt/wt) of oat extract and malt extract, inoculated with $2.5 \times 10^{7} \mathrm{cfu} / \mathrm{mL}$ of L. plantarum CCFM8661, and then incubated at $35^{\circ} \mathrm{C}$. The $\mathrm{pH}$ value, titration acidity, and viable cell counts during 48 -h fermentation at $35^{\circ} \mathrm{C}$ and 25 -d storage at $4^{\circ} \mathrm{C}$, were determined at different intervals. The results showed that the promotion effects of oat extract and malt extract on L. plantarum CCFM8661 growth rate in milk were much stronger than almond, walnut, sweet corn, peanut, and soybean meals. In addition, the stimulation effect of oat extract was associated with its concentration, and was much stronger than that of malt extract. Furthermore, viable counts and titration acidity of yogurt were gradually increased in the oat extract group, whereas viable counts were gradually decreased and titration acidity were slightly increased in the malt extract group during the 25 -d storage at $4^{\circ} \mathrm{C}$. Key words: Lactobacillus plantarum, fermented milk, starter culture, growth promotion

Received July 2, 2019.

Accepted December 19, 2019.

*Corresponding author: fenghang0427@126.com

\section{INTRODUCTION}

New yogurts with plant extracts are currently popular in the dairy market. As consumers are increasingly interested in healthy diets and wellness, with the expectation that food can help them be healthier or may even be capable of preventing illness, probiotic food products made via fermentation of milk, cereals, fruits, and vegetables are receiving attention from scientists as well as consumers (Gupta and Abu Ghannam, 2012). Lactobacillus plantarum, a versatile lactic acid bacterium, is encountered in a range of environmental niches, and is most frequently isolated from plant-based fermented foods as well as from meat, fish, and dairy products (Seddik et al., 2017). Lactobacillus plantarum is widely employed in food fermentation, with a long history and classification as "generally recognized as safe," and has "qualified presumption of safety" status (Behera et al., 2018). Lactobacillus plantarum has proven ability to survive gastric transit and colonize the gut, with apparent safety to the consumer (de Vries et al., 2006). Therefore, increasing probiotic potentials of L. plantarum have been reported, including cholesterollowering (Costabile et al., 2017), immunomodulatory (Kechaou et al., 2013), and antimicrobial functions, controlling body fat gain and reducing inflammation and other adverse effects typically associated with immune and intestinal improvement (Abdelazez et al., 2018). In addition, L. plantarum has potential abilities to alleviate multiple species of heavy metal toxicity, such as cadmium (Zhai et al., 2014), lead (Tian et al., 2012), copper (Tian et al., 2015), and aluminum (Yu et al., 2016). For instance, L. plantarum CCFM8661 has excellent antioxidative activity and protective effects against lead-induced toxicity. It could decrease the lead $\left(\mathrm{Pb}^{2+}\right)$ levels of blood and tissue and the majority of $\mathrm{Pb}^{2+}$ bound on the surface of bacterial cells (Yin et al., 2016). 
Lactobacillus plantarum strains have been characterized by their enzyme systems ( $\alpha$-amylase, esterase, lipase, $\alpha$-glucosidase, $\beta$-glucosidase, enolase, phosphoketolase, lactase dehydrogenase, and others) and bioactive compounds (bacteriocin, dipeptides, and other preservative compounds). They are easy to culture because of utilization of multiple carbon and nitrogen sources (Hedberg et al., 2008) and are widely used in fermented vegetable, cereal, meat, fish, dairy, and beverage products, and ethnic fermented foods (Behera et al., 2018). However, it has generally been considered that L. plantarum has low technological importance in standard milk processing (Quigley et al., 2013). Because it requires multiple amino acid and vitamin auxotrophs (Bringel and Hubert, 2003), L. plantarum with weak proteolytic ability was hard to grow in milk because of the lack of sufficient free amino acids and peptides to support its growth when cultured alone. In L. plantarum, complete whole-genome sequences have revealed that $\operatorname{prt} B, \operatorname{prt} H$, and $\operatorname{prt} R$, the cell envelope protease genes involved in primary protein degradation, were absent (Kleerebezem et al., 2003), along with scarce proteolytic activities (Georgalaki et al., 2017). However, many genes encoding intracellular peptidases were found for peptide degradation, which was a vital reason for $L$. plantarum as an adjunct culture in cheese manufacture, for accelerating ripening ( $\mathrm{Li}$ et al., 2017; Blaya et al., 2018).

Over the last decades, the health-promoting benefits of probiotics have attracted considerable attention. Hitherto, fermented dairy products (yogurt and cheese) have been recognized as the best matrix for the administration of probiotics (Georgieva et al., 2009). Recently, an increasing number of strains of L. plantarum with promising probiotic properties have been isolated from dairy-related sources. Therefore, some studies have made efforts to stimulate the growth and acidification of $L$. plantarum in milk using growthpromoter supplements at laboratory scale. Excellent sources of antioxidant vitamins, bioactive compounds, and minerals dramatically promoted the biomass yield of L. plantarum in skim and whole milk, such as 6 key amino acids and 1 purine required for $L$. plantarum STIII (Ma et al., 2016), glycine and dipeptide (Leu-Leu and Gly-Gly) for L. plantarum N4 (Saguir et al., 2008), $10 \%$ blackberry juice (Yang et al., 2014), and fermented carrot juice (Demir et al., 2006). Although previous studies have showed certain promotion of the growth and acidification of L. plantarum in milk, at present there are no practice standards and guidelines for commercial food-grade ingredients on pure $L$. plantarum strain application in yogurt preparations.

It is known that L. plantarum is commonly used as a starter culture in plant-based fermentation, and plant- based meals should stimulate the growth and acidification of L. plantarum in milk. In this study, the growth and acidification of $L$. plantarum in milk supplemented with commercial plant-based meals were investigated, and $\mathrm{pH}$, titration acidity, and viable cell counts during 48-h fermentation at $35^{\circ} \mathrm{C}$ were initially determined. Malt extract and oat extract with outstanding stimulation effects on L. plantarum were further studied. Additionally, the growth-promoting effects among different L. plantarum strains were compared. Subsequently, a yogurt fermented only by L. plantarum was successfully developed, and the viable counts and titration acidity of the yogurt at different intervals during 25-d storage at $4^{\circ} \mathrm{C}$ were determined.

\section{MATERIALS AND METHODS}

\section{Chemicals and Reagents}

The skim milk powder ( $0.8 \%$ fat, $33.4 \%$ protein, $54.1 \%$ lactose, $7.9 \%$ minerals, and $3.8 \%$ moisture) used for milk fermentation was purchased from Fonterra Ltd. (Auckland, New Zealand). Oat extract $[11.2 \%$ protein, $1.54 \%$ moisture, $1.64 \%$ sulfated ash, and dextrose equivalent (DE) value 30\%] and malt extract $(4.96 \%$ protein, $1.63 \%$ moisture, $1.75 \%$ sulfated ash, and DE value $47 \%$ ) were purchased from Döhler Food and Beverage Ingredients (Jinshan) Co. Ltd. (Shanghai, China). Almond (21.8\% protein, $19.2 \%$ fat, $1.76 \%$ ash, and $2.73 \%$ moisture), walnut (12.4\% protein, $39.1 \%$ fat, $29.4 \%$ carbohydrate, and $2.46 \%$ moisture), sweet corn $(2.8 \%$ protein, $1.4 \%$ fat, $83 \%$ carbohydrate, and $2.91 \%$ moisture), peanut ( $12.1 \%$ protein, $15.3 \%$ fat, $61.2 \%$ carbohydrate, and $2.89 \%$ moisture), and soybean (41.4\% protein, $21.6 \%$ fat, $28.0 \%$ carbohydrate, and $3.54 \%$ moisture) meals were purchased from Xuzhou Nature Biotechnology Co. Ltd. (Xuzhou, China). Phenolphthalein (98\%) was purchased from Canspec Inc. (Shanghai, China). All other chemicals were obtained from commercial sources and were of analytical grade.

\section{Bacterial Strains and Culture Conditions}

Lactobacillus plantarum CCFM8661, 8610, and 8742 were obtained from the Culture Collections of Food Microbiology, Jiangnan University (Wuxi, China). Lactobacillus plantarum ST-III was purchased from State Key Laboratory of Dairy Biotechnology (Shanghai, China). Before the experiments, these frozen stock cultures were inoculated in de Man, Rogosa, and Sharpe medium and transferred 3 times at $37^{\circ} \mathrm{C}$ for $16 \mathrm{~h}$, respectively. After culture, cells were harvested and centrifuged at $5,000 \times g$ for $5 \mathrm{~min}$ and washed twice with PBS (0.01 $M, \mathrm{pH}$ 7.4). The harvested cells were resuspended and 
concentrated with $10 \%$ (wt/vol) skim milk, which was sterilized in a YXQ-LS-70A autoclave (Shanghai Boxun Industry and Commerce Co. Ltd., Shanghai, China) at $108^{\circ} \mathrm{C}$ for $15 \mathrm{~min}$. The milk was subsequently dried in a Labconco 12L lyophilizer (Labconco Corp., Fort Scott, KS). The number of bacteria in the lyophilized preparations was determined via plate colony counting before later experiments.

\section{Preparation of Fermented Milk}

Fermented milk was prepared using the following procedure. Whole milk powder was reconstituted at a concentration of $200 \mathrm{~g} / \mathrm{L}$ and then mixed and stirred moderately with a mechanical stirrer (Eurostar 40, IKA Werke GmbH and Co., Staufen, Germany) at room temperature for $30 \mathrm{~min}$ to dissolve fully. The dispersion was then stored overnight at $4^{\circ} \mathrm{C}$ for complete hydration of casein micelles before use. Ingredients for improving the yogurt texture and flavor were blended initially and then gradually added to $60^{\circ} \mathrm{C}$ water, heated using a thermostated hotplate magnetic stirrer (C-MAG HS 7, IKA Werke). The volume of the water was equal to that of the reconstituted milk. The addition rates of sugar, plant-based meals, Novation 2300 modified starch (Ingredion China Ltd., Shanghai, China), low-methoxyl pectin, and natural whey were $70 \mathrm{~g} / \mathrm{L}, 75 \mathrm{~g} / \mathrm{L}, 5 \mathrm{~g} / \mathrm{L}, 1.5$ $\mathrm{g} / \mathrm{L}$, and $2 \mathrm{~g} / \mathrm{L}$, respectively. After fully dissolving, the prepared solution and reconstituted milk were mixed at $65^{\circ} \mathrm{C}$ and then homogenized by a 2-stage high-pressure GJJ-0.05/40 homogenizer (Shanghai Nuoni Light Industry Machinery Co. Ltd., Shanghai, China), using pressures around $15 \mathrm{MPa}$ for the first stage and $5 \mathrm{MPa}$ for the second stage, to break large fat globules down to smaller ones and prevent the separation of cream from milk (Geciova et al., 2002). The milk obtained $(60 \mathrm{~mL})$ was subpacked in 100-mL glass jars and then sterilized at $108^{\circ} \mathrm{C}$ for $15 \mathrm{~min}$ in a YXQ-LS-70A autoclave (Shanghai Boxun Industry and Commerce Co. Ltd.). We chose these sterilization conditions $\left(108^{\circ} \mathrm{C} / 15 \mathrm{~min}\right)$ because $L$. plantarum, with weak growth and acid production capacity in milk, could not inhibit the growth of the other microorganisms, such as spores of Bacillus, at low-temperature pasteurization $\left(80\right.$ to $\left.85^{\circ} \mathrm{C} / 15 \mathrm{~min}\right)$ in the laboratory (Halvorson and Church, 1957; Sadek et al., 2018), which would result in the deterioration of milk quality.

Proper cell concentrations of solution containing $L$. plantarum were prepared and inoculated, with the inoculum dose calculated as $2.0 \times 10^{7} \mathrm{cfu} / \mathrm{mL}$. The jars were shaken gently to distribute the starter and then incubated statically at $35^{\circ} \mathrm{C}$ in a BSP-150 biochemical incubator (Boxun Ltd., Shanghai, China) for $12 \mathrm{~h}$. Aliquots of samples were removed and collected at 2- to 4-h intervals for determination of titration acidity, $\mathrm{pH}$, and viable cell counts.

\section{Measurement of TA and $\mathrm{pH}$}

The $\mathrm{pH}$ of fermented milk at different intervals was measured with an OHAUS ST3100 digital pH-meter (Changzhou, China). The titration acidity was determined according to China National Food Safety Standard GB 5413.34-2010 (IDF, 1978, 1981). In brief, $10 \mathrm{~g}$ of sample was mixed with $20 \mathrm{~mL}$ of freshly boiled and cooled distilled water and then titrated with $0.1 \mathrm{~mol} / \mathrm{L}$ $\mathrm{NaOH}$ using $0.5 \%$ phenolphthalein as indicator until the color changed to pink and disappeared for $30 \mathrm{~s}$. The volume consumed by titration of $\mathrm{NaOH}$ standard solution was recorded, and then the titration acidity (degrees Thorner, ${ }^{\circ} \mathbf{T}$ ) of sample was calculated according to the following equation (Jia et al., 2016):

$$
{ }^{\circ} \mathrm{T}=c \times v \times 100 /(m \times 0.1),
$$

where $c$ is the molarity of $\mathrm{NaOH}$ standard solution; $v$ is the volume consumed by titration of $\mathrm{NaOH}$ standard solution; and $m$ is the mass of the fermented milk sample.

\section{Plate Count}

The viable counts of cells in the milk during fermentation were determined using the plate count method. In detail, $10 \mathrm{~g}$ of each sample at 0-, 2-, 4-, 6-, 8-, 10-, and 12-h intervals after inoculation were aseptically collected and diluted with $90 \mathrm{~mL}$ of $0.85 \%$ (wt/vol) sterile saline solution and then serially diluted 10-fold to appropriate concentrations. One milliliter of diluted solution was pipetted into aseptic Petri dishes, and then the de Man, Rogosa, and Sharpe agar culture medium, melted and cooled to proper temperature, was poured into dishes and shaken. After the medium cooled and solidified, these plates were inverted and incubated in a BSP-150 biochemical incubator (Boxun Ltd., Shanghai, China) at $35^{\circ} \mathrm{C}$ for $48 \mathrm{~h}$. The numbers of L. plantarum were expressed as colony forming units per milliliter. The stimulation effects of malt extract and oat extract were compared by growth curve parameters. To investigate the shelf life of the fermented milk, the titration acidity and viable counts of the yogurt at different intervals were determined during $25 \mathrm{~d}$ of storage at $4^{\circ} \mathrm{C}$.

\section{Kinetics of Growth Curves}

In general, when the bacteria growth curve is defined as the logarithm of the viable cell number plotted against time, the growth rate changes and results in a 
sigmoidal curve, with a lag phase before the beginning of exponential growth. After inoculation $\left(N_{0}\right)$, growth rate often starts at a value of 0 and then accelerates to a maximal value $\left(\mu_{m}\right)$ in a certain period of time. At the end of the exponential phase, the growth rate gradually decreases and finally reaches 0 , so that an asymptote $\left(N_{\max }\right)$ is reached. The maximum specific growth rate $\left(\mu_{m}\right)$ is a valuable parameter of the growth curve, which can illustrate the stimulation effects on $L$. plantarum by supplemented plant-based meals with different concentrations. The logistics equation (Chowdhury et al., 2007) was usually selected and fitted to growth data by nonlinear regression according to equation [2] with a Levenberg-Marquardt algorithm:

$$
\log _{10} N_{t}=A+B /\left\{1+\exp \left[-\mu_{m} \times\left(t-t_{m}\right)\right]\right\},
$$

where $\log _{10} N_{t}$ is the number of viable cells at the observed time $(t)$ during the different growth phases after the initial inoculation; $A$ is the asymptotic log count as $t$ decreases indefinitely (i.e., $\log _{10} N_{0}$ ); $B$ is the asymptotic amount of growth as $t$ increases indefinitely; $\mu_{m}$ is the maximum specific growth rate at $t_{m}$ during the exponential phase; and $t_{\mathrm{m}}$ is the time at which the maximum specific growth rate was achieved (Fujikawa et al., 2004). For each curve of this experiment, $A, B$, $\mu_{m}$, and $t_{m}$ can be calculated after data fitting under different experiment conditions. After data fitting, the parameters $\log _{10} N_{t}, \mu_{m}, t_{m}, A$, and $B$ were derived from each curve. The obtained parameters of growth curve were further analyzed, to study the stimulation effects of malt extract and oat extract at different concentrations.

\section{Statistical Analysis}

All data were expressed as means and standard deviation (SD) from 3 replicates. All statistical analyses were performed using the Statistical Analysis Software (SAS 9.2, SAS Institute Inc., Cary, NC). The confidence level was set at $P<0.05$ for statistical significance. Origin 8.0 software (OriginLab Corp., Northampton, MA) was selected for data plotting.

We selected 1stOpt1.5 PRO Software (7D-Soft High Technology Inc., Beijing, China) for data fitting, using Levenberg-Marquardt and universal global optimization (UGO) algorithms (Zhang et al., 2013). The reliability of the logistics equations describing the growth of $L$. plantarum in milk was determined via root mean square error (RMSE), sum of squares (SSE), and coefficient of determination $\left(\mathbf{R}^{2}\right)$ analysis. $\mathbf{R}^{2}$ is defined as the ratio of explained variation to total variation and is a measure of the degree of fit.

\section{RESULTS AND DISCUSSION}

\section{Stimulation Effects of Different Plant-Based Meals}

The acidification and growth of $L$. plantarum CCFM8661 in milk supplemented with plant-based meals at different intervals are shown in Figure 1. The $\mathrm{pH}$ value of fermented milk is shown in Supplemental Figure S1 (https://doi.org/10.3168/jds.2019-17200). Lactobacillus plantarum CCFM8661 grew significantly in the milk supplemented with plant-based meals compared with the control group without supplementation $(P<0.05)$. Additionally, in group 6 , the titration acidity reached $104 \pm 2^{\circ} \mathrm{T}(\mathrm{pH} 3.82)$ and $117 \pm 2^{\circ} \mathrm{T}(\mathrm{pH}$ 3.70 ), and the logarithms of viable counts reached 9.04 and $8.92 \mathrm{cfu} / \mathrm{g}$ at 24 and $48 \mathrm{~h}$, respectively. In group 7, the titration acidity was $90 \pm 1^{\circ} \mathrm{T}(\mathrm{pH} 3.95)$ and 112 $\pm 2^{\circ} \mathrm{T}$ (3.73), and the logarithms of viable counts were 9.08 and $9.04 \mathrm{cfu} / \mathrm{g}$ at 24 and $48 \mathrm{~h}$, respectively. The titration acidity and viable counts in groups 6 and 7 were much higher than other groups at the observed intervals $(P<0.05)$.

Similar promotive effects on growth were observed in experiments supplemented with adenine, amino acids, and blackberry juice for other L. plantarum strains (STIII, WCFS1, and LPHS; Ma et al., 2016; Yang et al., 2014). The logarithms of viable counts of $L$. plantarum in fermented milk supplemented with $0.1 \mathrm{~g} / \mathrm{L}$ adenine and $0.2 \mathrm{~g} / \mathrm{L}$ amino acids (Ile, Leu, Val, Tyr, Met, and Phe) were 8.34 and $9.18 \mathrm{cfu} / \mathrm{g}$ at 24 and $48 \mathrm{~h}$, respectively. In contrast, the logarithms of viable counts in control group were only 7.65 and $7.59 \mathrm{cfu} / \mathrm{g}$ at 24 and $48 \mathrm{~h}$, respectively. The $\mathrm{pH}$ values of cultures with the L. plantarum ST-III strain in the supplemented group were 5.11 and 4.08 at 24 and $48 \mathrm{~h}$, respectively. However, the $\mathrm{pH}$ values of the control group were only 6.06 and 5.77 at 24 and $48 \mathrm{~h}$, respectively. The $\mathrm{pH}$ values of fortified fermented milk with the L. plantarum WCFS1 strain and the control group were 4.89 and 5.87 at 24 $\mathrm{h}$, respectively. As for the L. plantarum LPHS strain, the $\mathrm{pH}$ value of fortified fermented milk was 5.00, and that of control group was only 5.97 at $24 \mathrm{~h}$. Moreover, we found that the growth of L. plantarum was significantly stimulated by blackberry juice in skim milk, and the logarithms of viable counts in fermented milk with blackberry juice were 10.02 and $11.3 \mathrm{cfu} / \mathrm{g}$ at 24 and $48 \mathrm{~h}$, respectively; whereas the logarithms of viable cell counts in the control group were 6.93 and $7.72 \mathrm{cfu} / \mathrm{g}$ at 24 and $48 \mathrm{~h}$, respectively.

According to manufacturer's instructions, malt extract is prepared from barley malt and barley, and the barley malt is treated with hot water extraction, saccharification by amyloglucosidase and glucose isomerase, filtration, concentration, and vacuum drying. 

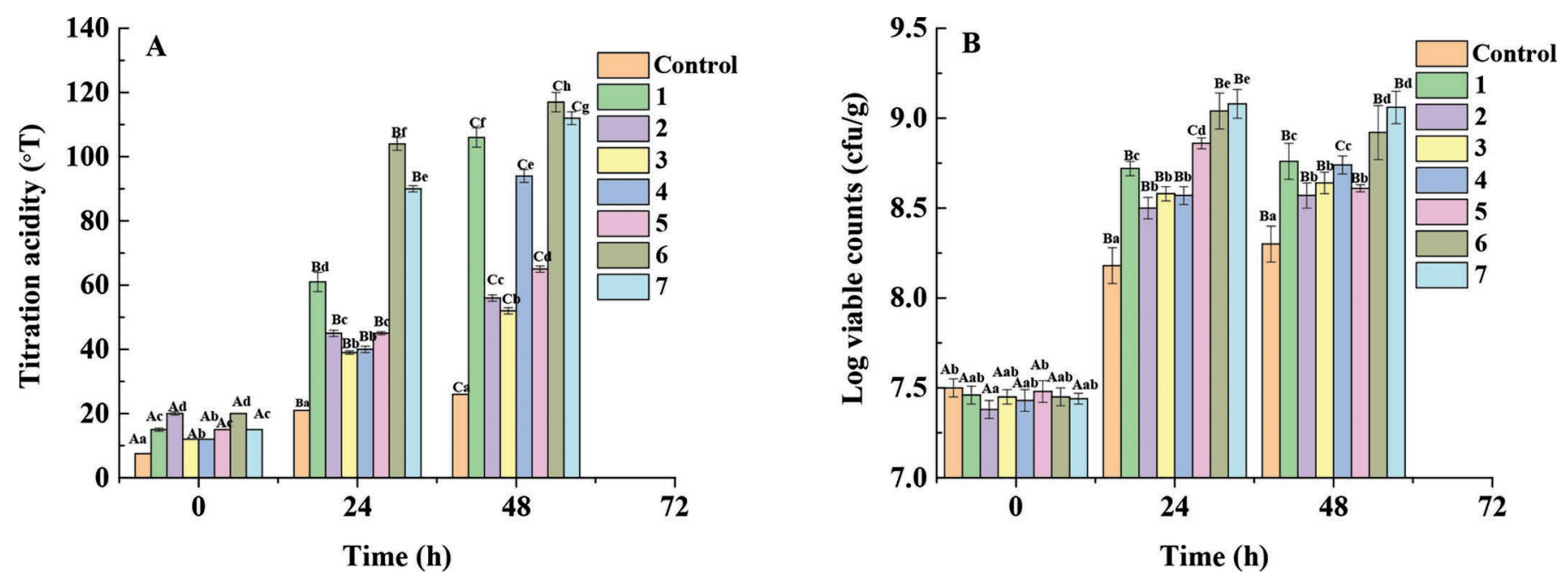

Figure 1. The acidification (A) and growth (B) of Lactobacillus plantarum CCFM8661 in milk supplemented with different plant-based meals. The addition rates were $5 \%$ (wt/wt) for all meals. Control group was unsupplemented milk; groups 1-7 were milk supplemented with almond meal, walnut meal, sweet corn meal, peanut meal, soybean meal, malt extract, and oat extract, respectively. Columns are shown as mean $\pm \mathrm{SD}$. Within each group, means with different lowercase letters were significantly different $(P<0.05)$ among different groups. Within each time period, means with different capital letters were significantly different among different time points $(P<0.05)$.

Malting is basically the germination process carried out under controlled conditions (Østergaard et al., 2002), during which several seed biopolymers, including the storage proteins, must be hydrolyzed. The barley proteins are initially solubilized by endoproteases and then further degraded by exopeptidases (Jones, 2005). Oat extract is prepared from the enzymatic pretreatments of oat flour, protease, and $\beta$-glucanase, derived from bacteria or fungi, followed by filtration, concentration, and vacuum drying. Whether endogenous or exogenous proteases, proteins in barley and oat meal are partially hydrolyzed and thus provide peptides needed for growth of L. plantarum.

Although the stimulation effects of malt extract and oat extract on the growth of $L$. plantarum in milk were not as great as those of blackberry juice, growth rates from malt and oat extracts were much faster than those of supplementation with $0.1 \mathrm{~g} / \mathrm{L}$ adenine and $0.2 \mathrm{~g} / \mathrm{L}$ amino acids. In addition, as food-grade ingredients, malt extract and oat extract have many functional aspects. For instance, oat extract contains relatively hydrophilic phenolic compounds, including avenanthramides and tocopherol, which have very obvious antioxidant abilities, known to protect the body from damage by free radicals. The antioxidants in oat extract can inhibit the oxidation of low-density lipoproteins (LDL), prevent development of atherosclerosis, and decrease levels of LDL cholesterol, reducing risks of cardiovascular diseases such as coronary heart disease (Gray et al., 2002). Malt extract also has obvious antioxidant activity and anti-aging effects; it can prevent the decrease of antioxidant enzyme activity and reduce the contents of malondialdehyde and carbonyl groups in liver and brain tissues, to prevent cancer, inflammation, Parkinson's disease, and other health problems (Yang et al., 2010). Therefore, the stimulation effects of malt extract and oat extract on L. plantarum in milk fermentation need to be further investigated in greater detail.

\section{Milk Fermentation Supplemented with Malt Extract and Oat Extract}

The acidification and growth of L. plantarum CCFM8661 in milk supplemented with different concentrations $(3.5 \%, 5 \%, 7.5 \%$, and $10 \%)$ of malt extract and oat extract during 12-h fermentation, which were measured at 2-h intervals, are shown in Figure 2. The $\mathrm{pH}$ values of fermented milk are presented in Supplemental Figure S2 (https://doi.org/10.3168/jds.2019-17200). Compared with the control group, both malt extract and oat extract significantly stimulated the acidification and growth of L. plantarum CCFM8661 in milk $(P<0.05)$. The stimulation effects on $L$. plantarum CCFM8661 were associated with addition rates. The titration acidities of fermented milk with malt and oat extracts at $10 \mathrm{~h}$ were between 45 and $85^{\circ} \mathrm{T}$. The rates of acidification and growth of L. plantarum CCFM8661 in oat extract were much stronger than those of malt extract, which might be due to the higher protein content and greater levels of peptides in oat extract, compared with malt extract. In the case of oat extract, the stimulation effects on L. plantarum CCFM8661 were dependent on the addition rates. However, in the case of malt extract, the stimulation effects as func- 
tions of addition rates were not very linear, with the optimal concentration at $7.5 \%$. The titration acidities of fermented milk were highly associated with viable cell counts; the higher the titration acidity achieved, the greater viable counts were obtained in the sample.

As the curves grew exponentially, the experimental data were fitted with the formula of logistic equation [2], using Levenberg-Marquardt and UGO algorithms, to describe the sigmoidal growth of viable cells. 1stOpt1.5 PRO software is an optimization analysis software package for mathematics with independent intellectual property rights. Compared with Matlab, Origin Pro, SAS, SPSS, and other software packages, 1stOpt can enable calculations to achieve the optimal convergence without providing initial values of parameters. Due to its strong optimization and fault tolerance abilities in most situations, this software is widely used in various fields of scientific research, in problems of nonlinear regression, curve fitting, calculation of nonlinear model parameters, and linear and nonlinear programming ( $\mathrm{He}$ and $\mathrm{Yu}, 2013)$.

These simplified assumptions were indicated with fixed values, to account for the slight deviations of the model from the data. The model of logistic curve was proposed to simulate experimental data with variable parameter $\log _{10} N_{t}$ and $t_{m}$, and the parameter $\mu_{m}$ represented the maximum specific growth rate. It has been previously observed in experiment that growth rate varies with the addition of different concentrations of malt extract and oat extract. As shown in Table 1, the
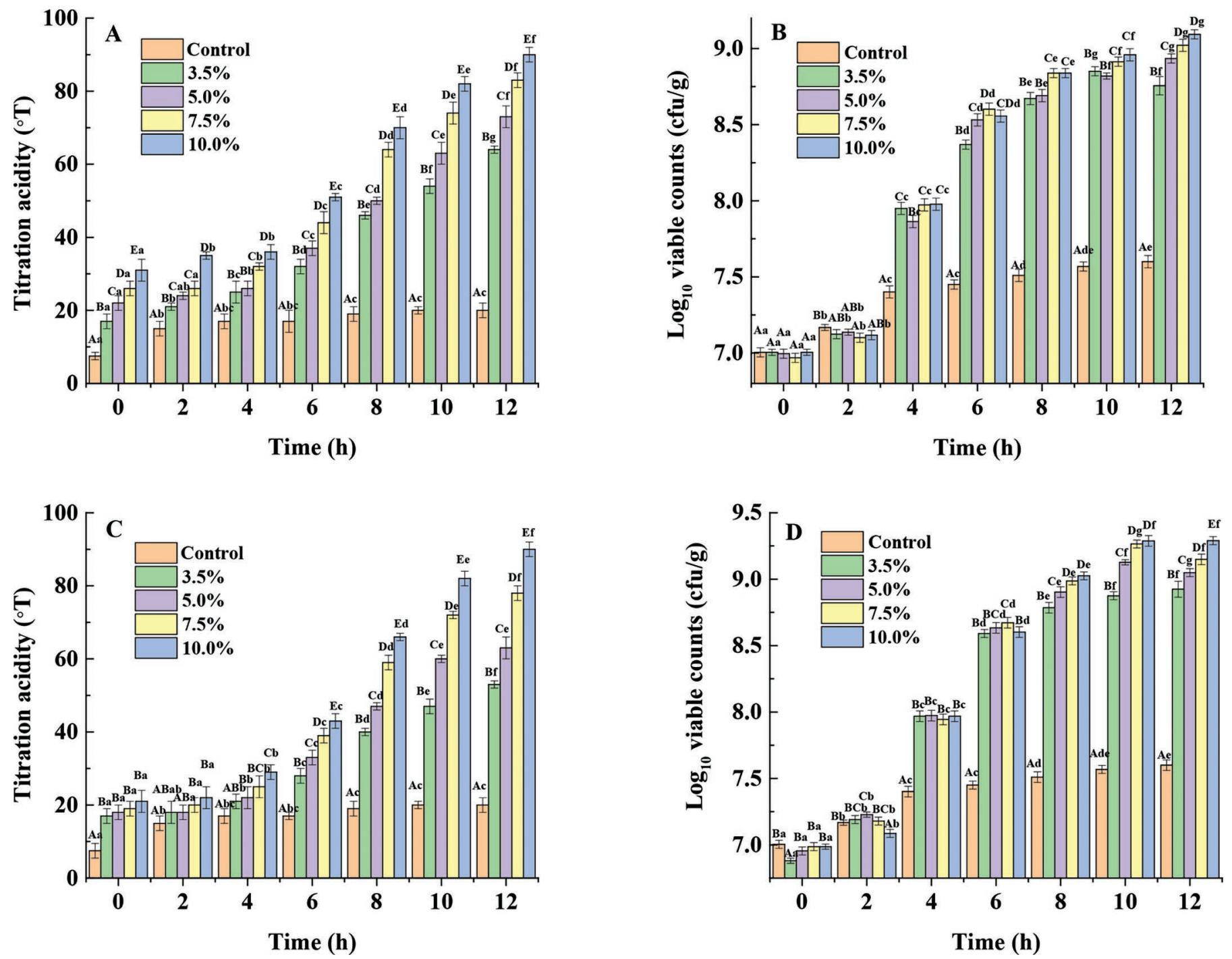

Figure 2. Milk fermentation supplemented with different concentrations of malt extract (A, B) and oat extract (C, D). Error bars represent SD. ${ }^{\circ} \mathrm{T}=$ degrees Thorner. Columns are shown as mean \pm SD. Within each time period, means with different lowercase letters are significantly different $(P<0.05)$ among different time points. Within each group, means with different capital letters are significantly different among different groups $(P<0.05)$. 
Table 1. Results of parameters of Lactobacillus plantarum 8661 growth curves in milk supplemented with different concentrations of malt and oat extracts fitting by logistic model ${ }^{1}$

\begin{tabular}{|c|c|c|c|c|c|c|c|c|c|c|c|c|c|c|}
\hline Addition rate & \multicolumn{7}{|c|}{ L. plantarum CCFM 8661 with malt } & \multicolumn{7}{|c|}{ L. plantarum CCFM 8661 with oat } \\
\hline $3.50 \%$ & 6.853 & 1.936 & 0.741 & 3.925 & 0.0739 & 0.0382 & 0.989 & 6.684 & 2.227 & 0.668 & 3.668 & 0.0299 & 0.0063 & 0.999 \\
\hline $5.00 \%$ & 6.885 & 1.946 & 0.800 & 4.058 & 0.0801 & 0.0449 & 0.988 & 6.807 & 2.279 & 0.699 & 3.998 & 0.0397 & 0.0110 & 0.998 \\
\hline $7.50 \%$ & 6.860 & 2.086 & 0.869 & 3.974 & 0.0552 & 0.0214 & 0.995 & 6.859 & 2.341 & 0.728 & 4.289 & 0.0552 & 0.0213 & 0.996 \\
\hline $10.00 \%$ & 6.842 & 2.173 & 0.733 & 4.092 & 0.0711 & 0.0353 & 0.992 & 6.788 & 2.518 & 0.650 & 4.441 & 0.0732 & 0.0375 & 0.994 \\
\hline
\end{tabular}

${ }^{1} A=$ asymptotic log count as time $(t)$ decreases indefinitely; $B=$ asymptotic amount of growth as $t$ increases indefinitely; $\mu_{m}=$ maximum specific growth rate at $t_{m}$ during the exponential phase; $t_{m}=$ time at which maximum specific growth rate was achieved; RMSE $=$ root mean square error; SSE $=$ sum of squares.

values of $\mu_{m}$ of different addition rates $(0 \%, 3.5 \%, 5 \%$, $7.5 \%$ ) were $0.732,0.741,0.800$, and 0.869 and 0.628 , $0.668,0.699$, and 0.728 with malt and oat, respectively, showing positive growth trends with addition of malt and oat extracts. In Figure 2B and 2D, the tendency of the experimental data coincided with the hypothesized growth and rose slowly, which could demonstrate that kinetic growth of L. plantarum CCFM8661 was influenced by the addition rates of malt and oat extracts. However, when the addition rate increased to $10 \%$, the value of $\mu_{m}$ decreased slightly along with counts of viable cells, which was suspected to be caused by the growth limit of L. plantarum CCFM8661. Similarly, with the same inoculum size, the calculated parameter $A$ was very close, and the parameter $B$, which referred to the asymptotic amount of growth with indefinite time, increased according to the same rule of different addition rates of malt and oat extracts (Table 1). In conclusion, the parameter of the equation showed the growth specificity of probiotics, and the stimulation effects $\left(\mu_{m}\right)$ on L. plantarum CCFM8661 were found to be positively associated with addition rates of malt and oat extracts.

The reliability and precision of the equations were determined through RMSE, SSE, and $\mathrm{R}^{2}$ analysis (Zhang et al., 2018). A measure of the degree of fit, $\mathrm{R}^{2}$ is defined as the ratio of explained variation to total variation. We used RMSE and SSE to measure the deviation between observed values and predicted values. Low dispersion between the experimental and predicted values, which indicates the higher precision and greater fit of the logistic model, can be illustrated by the low values of RMSE and SSE and high value of $R^{2}$. In all experiments with addition of malt and oat extracts, we found the maximum values of RMSE and SSE to be 0.0801 and 0.0449 , respectively. The logistic equation exhibited high $\mathrm{R}^{2}$ values, ranging from 0.971 to 0.999 . These low RMSE and SSE values and high $R^{2}$ values indicate the goodness of fit of the logistic model (Taheri Garavand et al., 2011). Therefore, the logistic model could be used to describe the growth of $L$. plantarum strains in fermented milk.

\section{Stimulation Effects Among Different L. plantarum Strains}

The growth of L. plantarum strains in fermented milk supplemented with $7.5 \%$ malt and oat extracts during 12-h incubation, at 4-h intervals, are shown in Figure 3 . The study was performed in multiple groups, and the stimulation effects on growth were analyzed at time points of $0,4,8$, and $12 \mathrm{~h}$ among 4 kinds of L. plantarum strains, including ST-III, CCFM8661, CCFM8610, and CCFM8742.

In this experiment, the logarithms of viable counts of L. plantarum CCFM8661 in fermented milk were calculated and analyzed to significantly define the stimulation effects among different L. plantarum strains. As shown in Figure 3, after inoculation of L. plantarum 8661 with malt and oat extracts, the logarithms of viable counts at intervals of $0,4,8,12$ were $6.97,7.97$, 8.84 , and $9.02 \mathrm{cfu} / \mathrm{g}$ in the malt extract group and 6.99, $7.94,8.99$, and $9.15 \mathrm{cfu} / \mathrm{g}$ in the oat extract group, respectively. We see that the number of viable cells increased with the fermentation time, and the growth rate was first increased to $\mu_{m}$, then gradually decreased to maintain stability after inoculation.

In Figure 3, the growth rates of 4 L. plantarum strains in milk supplemented with malt and oat extracts increased obviously compared with the control group ( $P$ $<0.05$ ), which illustrates that the growth in milk of these L. plantarum strains could be definitely stimulated by malt and oat extracts. Interestingly, we discovered a difference among the $4 \mathrm{~L}$. plantarum strains in the growth in the milk supplemented with malt extract and oat extract. In addition, ST-III had weaker growth with oat extract than with malt extract $(P<0.05)$, which was not observed in the other 3 strains. Among the $4 \mathrm{~L}$. plantarum strains, the highest logarithms of viable counts were $9.02 \mathrm{cfu} / \mathrm{g}$ of $L$. plantarum 8661 

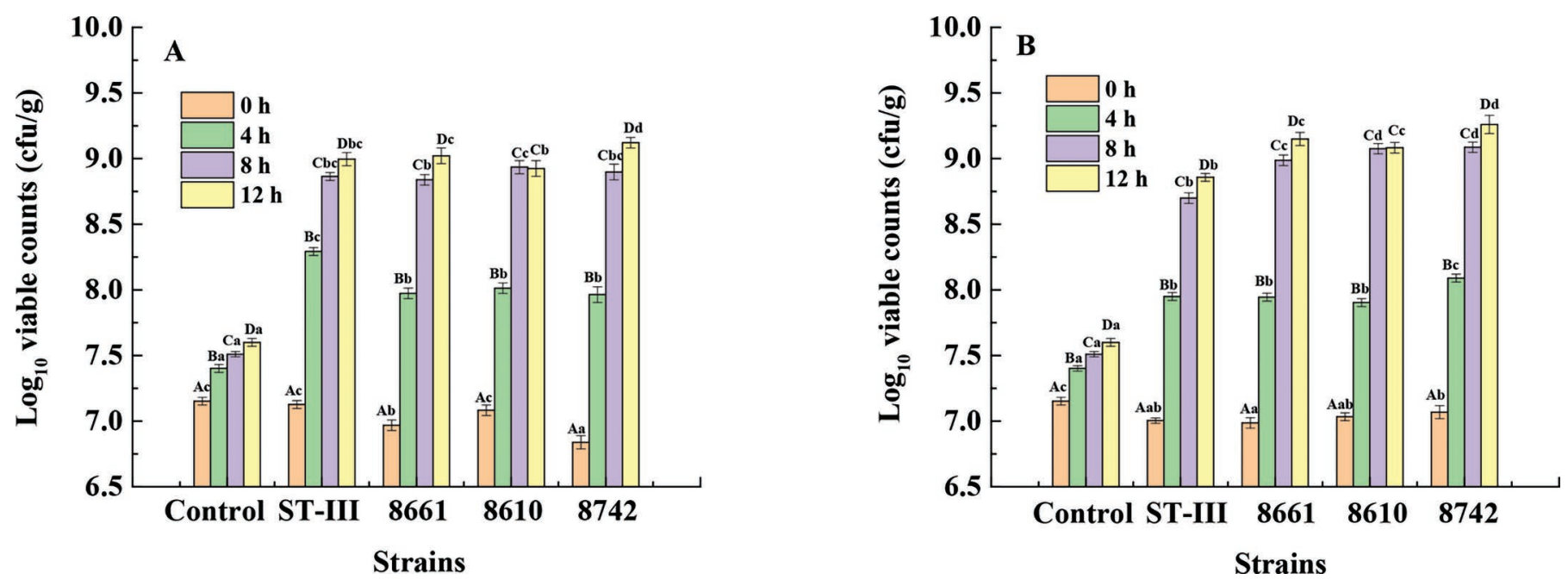

Figure 3. The growth of control and Lactobacillus plantarum ST-III, 8661, 8610, and 8742 with 7.5\% malt extract (A) and oat extract (B) at time points of $0,4,8$, and $12 \mathrm{~h}$. The control group was defined as logarithm of viable counts of strain 8661, which is the main target strain of this article, at different time intervals $(0,4,8$, and $12 \mathrm{~h})$ without the addition of malt and oat extracts. Columns are shown as mean \pm SD Within each time period, means with different lowercase letters are significantly different $(P<0.05)$ among different time points. Within each group, means with different capital letters are significantly different among different groups $(P<0.05)$.

and $9.12 \mathrm{cfu} / \mathrm{g}$ of L. plantarum 8742 with malt extract, and $9.15 \mathrm{cfu} / \mathrm{g}$ of L. plantarum 8661 and $9.26 \mathrm{cfu} / \mathrm{g}$ of L. plantarum 8742 with oat extract. In contrast, the logarithm of viable counts was only $7.64 \mathrm{cfu} / \mathrm{g}$ in the control group when the incubation time reached $12 \mathrm{~h}$. Therefore, it can be determined that L. plantarum 8742 and 8661 both have better growth abilities than those of L. plantarum ST-III and 8610.

On the basis of the experiment data analyzed above, the fermented milk with the addition of malt and oat extracts can be verified to effectively stimulate the growth of L. plantarum strains. In this sense, to achieve higher cell viability of $L$. plantarum strains, this method can be transferred to the industrial production of fermented milk with varieties of $L$. plantarum strains, to produce more effectiveness of $L$. plantarum strains in the functional food industry. However, because the severe thermal treatment applied in our experiment $\left(108^{\circ} \mathrm{C} / 15 \mathrm{~min}\right)$ possibly causes greater nutritional loss in the milk than do those used in industrial thermal processes (that is, pasteurization and UHT treatment), the critical nutrients in malt and oat extracts supporting the substantial growth of L. plantarum in milk still need to be further studied.

\section{Shelf Life of Yogurt Fermented by L. plantarum CCFM8661}

Milk supplemented with $7.5 \%$ malt extract and oat extract was fermented for $8 \mathrm{~h}$ after inoculation and then rapidly cooled and stored in a refrigerator at $4^{\circ} \mathrm{C}$. The titration acidity and viable counts of fermented milk supplemented with $7.5 \%$ malt extract and oat extract during 25-d storage are shown in Figure 4. At the end of fermentation, the titration acidities of milk containing malt extract and oat extract were $72 \pm 2^{\circ} \mathrm{T}$ and $67 \pm 1^{\circ} \mathrm{T}$, respectively. The slightly higher titration acidity for the malt extract group might be due to the higher initial titration acidity and faster growth of

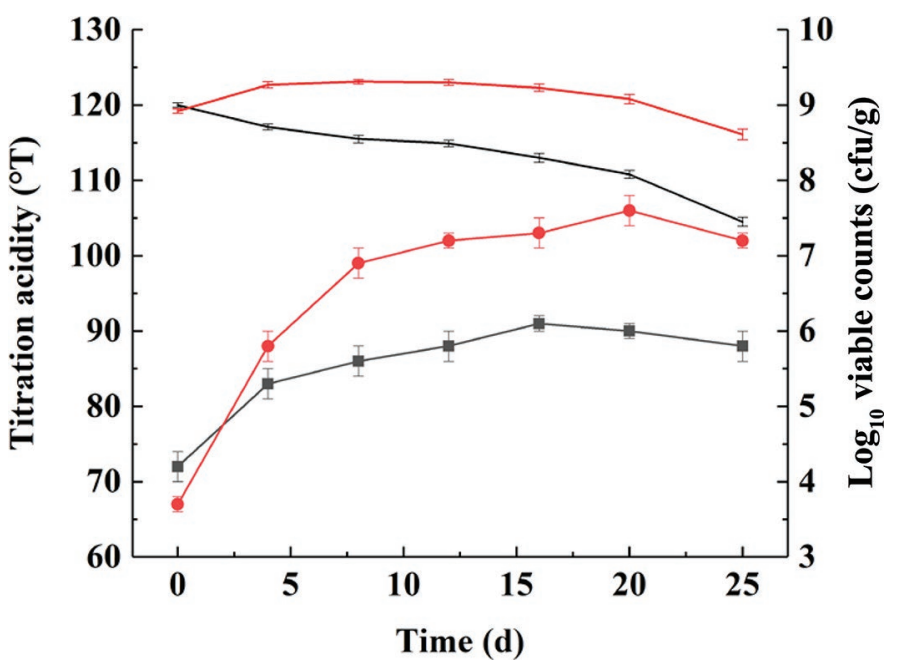

Figure 4. The titration acidity $\left({ }^{\circ} \mathrm{T}=\right.$ degrees Thorner $)$ and viable cell counts of fermented milk supplemented with $7.5 \%$ malt extract and oat extract during 25-d refrigerated storage. Error bars represent SD. The solid lines with solid squares (ם) and solid circles (-) represent the titration acidity of fermented milk containing malt extract and oat extract, respectively; the gray line and red line depict the viable counts in fermented milk containing malt extract and oat extract, respectively. 
L. plantarum CCFM8661 (Figure 2). Interestingly, the post-acidification of the fermented milk containing oat extract was much stronger than that of the malt extract group, which coincided with the continuous growth of this strain during the first 12-d storage and then slowly declined. However, the viable counts in fermented milk containing malt extract gradually decreased, which might be due to the depletion of available nitrogen sources, so that they could not sustain the growth. At the end of 25-d storage, the logarithms of viable counts in fermented milk supplemented with malt extract and oat extract were $7.45 \mathrm{cfu} / \mathrm{g}$ and $8.61 \mathrm{cfu} / \mathrm{g}$, respectively.

For application of malt extract and oat extract alone as growth promoters of $L$. plantarum in yogurt preparation, the weakness of post-acidification and reduction of viable cells are now being investigated. Therefore, it is very valuable to study the combination of these 2 components and to reduce the adverse effects of postacidification and viable count reduction by themselves.

\section{CONCLUSIONS}

In conclusion, the investigated plant-based meals have different promotion effects on the growth of $L$. plantarum CCFM8661 in milk compared with control. The promotion effects of malt extract and oat extract on CCFM8661 growth rates in milk were much stronger than those of the other meals. The stimulation effects of malt extract and oat extract were associated with their addition rates. Furthermore, the stimulation effect of oat extract was much stronger than that of malt extract. We also found certain differences in the growth among different $L$. plantarum strains in milk supplemented with malt extract and oat extract. As widely used food-grade ingredients, malt and oat extracts can be applied in the development of novel probiotic yogurt and yogurt drink using only L. plantarum strains or coupled with other lactic acid bacterial strains. Extensive research is needed to study the critical nutrients in malt and oat extracts that support the substantial growth of L. plantarum in milk.

\section{ACKNOWLEDGMENTS}

This work was supported by the National Natural Science Foundation of China (No. 31771953; Beijing), the National First-Class Discipline Program of Food Science and Technology (JUFSTR20180102, Wuxi City, China), and the Collaborative Innovation Center of Food Safety and Quality Control in Jiangsu Province (Wuxi, China). The authors have not stated any conflicts of interest.

\section{REFERENCES}

Abdelazez, A., H. Abdelmotaal, Z. Zhu, J. Fang, R. Sami, L. Zhang, A. R. Al Tawaha, and X. Meng. 2018. Potential benefits of Lactobacillus plantarum as probiotic and its advantages in human health and industrial applications: A review. Adv. Environ. Biol. 12:16-27.

Behera, S. S., R. C. Ray, and N. Zdolec. 2018. Lactobacillus plantarum with functional properties: An approach to increase safety and shelf-life of fermented foods. BioMed Res. Int. 2018:9361614. https: //doi.org/10.1155/2018/9361614

Blaya, J., Z. Barzideh, and G. LaPointe. 2018. Symposium review: Interaction of starter cultures and nonstarter lactic acid bacteria in the cheese environment. J. Dairy Sci. 101:3611-3629. https:// doi.org/10.3168/jds.2017-13345.

Bringel, F., and J. C. Hubert. 2003. Extent of genetic lesions of the arginine and pyrimidine biosynthetic pathways in Lactobacillus plantarum, L. paraplantarum, L. pentosus, and L. casei: Prevalence of $\mathrm{CO}_{2}$-dependent auxotrophs and characterization of deficient $\arg$ genes in L. plantarum. Appl. Environ. Microbiol. 69:2674-2683. https://doi.org/10.1128/AEM.69.5.2674-2683.2003.

Chowdhury, B. R., R. Chakraborty, and U. R. Chaudhuri. 2007. Validity of modified Gompertz and logistic models in predicting cell growth of Pediococcus acidilactici $\mathrm{H}$ during the production of bacteriocin pediocin AcH. J. Food Eng. 80:1171-1175. https://doi .org/10.1016/j.jfoodeng.2006.08.019.

Costabile, A., I. Buttarazzi, S. Kolida, S. Quercia, J. Baldini, J. R. Swann, P. Brigidi, and G. R. Gibson. 2017. An in vivo assessment of the cholesterol-lowering efficacy of Lactobacillus plantarum ECGC 13110402 in normal to mildly hypercholesterolaemic adults. PLoS One 12:e187964. https://doi.org/10.1371/journal .pone.0187964.

de Vries, M. C., E. E. Vaughan, M. Kleerebezem, and W. M. de Vos. 2006. Lactobacillus plantarum - Survival, functional and potential probiotic properties in the human intestinal tract. Int. Dairy J. 16:1018-1028. https://doi.org/10.1016/j.idairyj.2005.09.003.

Demir, N., K. S. Bahçeci, and J. Acar. 2006. The effects of different initial Lactobacillus plantarum concentrations on some properties of fermented carrot juice. J. Food Process. Preserv. 30:352-363. https://doi.org/10.1111/j.1745-4549.2006.00070.x.

Fujikawa, H., A. Kai, and S. Morozumi. 2004. A new logistic model for Escherichia coli growth at constant and dynamic temperatures. Food Microbiol. 21:501-509. https://doi.org/10.1016/j.fm.2004.01 .007 .

Geciova, J., D. Bury, and P. Jelen. 2002. Methods for disruption of microbial cells for potential use in the dairy industry-A review. Int. Dairy J. 12:541-553. https://doi.org/10.1016/S0958 -6946(02)00038-9.

Georgalaki, M., G. Zoumpopoulou, E. Mavrogonatou, G. Van Driessche, V. Alexandraki, R. Anastasiou, M. Papadelli, M. Kazou, E. Manolopoulou, D. Kletsas, B. Devreese, K. Papadimitriou, and E. Tsakalidou. 2017. Evaluation of the antihypertensive angiotensinconverting enzyme inhibitory (ACE-I) activity and other probiotic properties of lactic acid bacteria isolated from traditional Greek dairy products. Int. Dairy J. 75:10-21. https://doi.org/10.1016/j .idairyj.2017.07.003.

Georgieva, R., I. Iliev, T. Haertlé, J. Chobert, I. Ivanova, and S. Danova. 2009. Technological properties of candidate probiotic Lactobacillus plantarum strains. Int. Dairy J. 19:696-702. https://doi.org/ 10.1016/j.idairyj.2009.06.006.

Gray, D. A., M. J. Clarke, C. Baux, J. P. Bunting, and A. M. Salter. 2002. Antioxidant activity of oat extracts added to human LDL particles and in free radical trapping assays. J. Cereal Sci. 36:209218. https://doi.org/10.1006/jcrs.2001.0456.

Gupta, S., and N. Abu Ghannam. 2012. Probiotic fermentation of plant based products: Possibilities and opportunities. Crit. Rev. Food Sci. Nutr. 52:183-199. https://doi.org/10.1080/10408398 .2010.499779.

Halvorson, H., and B. Church. 1957. Biochemistry of spores of aerobic bacilli with special reference to germination. Bacteriol. Rev. 21:112-131. https://doi.org/10.1128/MMBR.21.2.112-131.1957. 
He, Z., and H. Yu. 2013. Data fusion based on first optimization and its comparison with the traditional algorithms. Pages 1432-1436 in Proc. 2013 6th International Congress on Image and Signal Processing. 6th International Congress on Image and Signal Processing, Hangzhou, China.

Hedberg, M., P. Hasslöf, I. Sjöström, S. Twetman, and C. StecksénBlicks. 2008. Sugar fermentation in probiotic bacteria-An in vitro study. Oral Microbiol. Immunol. 23:482-485. https://doi.org/10 .1111/j.1399-302X.2008.00457.x.

IDF. 1978. International Dairy Federation standard IDF 86:1978 dried milk-Determination of titratable acidity (reference method).

IDF. 1981. International Dairy Federation standard IDF 81:1981 Dried milk-Determination of titratable acidity (routine method).

Jia, R., H. Chen, H. Chen, and W. Ding. 2016. Effects of fermentation with Lactobacillus rhamnosus GG on product quality and fatty acids of goat milk yogurt. J. Dairy Sci. 99:221-227. https://doi .org/10.3168/jds.2015-10114.

Jones, B. L. 2005. Endoproteases of barley and malt. J. Cereal Sci. 42:139-156. https://doi.org/10.1016/j.jcs.2005.03.007.

Kechaou, N., F. Chain, J. J. Gratadoux, S. Blugeon, N. Bertho, C. Chevalier, R. Le Goffic, S. Courau, P. Molimard, J. M. Chatel, P. Langella, and L. G. Bermúdez Humarán. 2013. Identification of one novel candidate probiotic Lactobacillus plantarum strain active against influenza virus infection in mice by a large-scale screening. Appl. Environ. Microbiol. 79:1491-1499. https://doi.org/10.1128/ AEM.03075-12.

Kleerebezem, M., J. Boekhorst, R. van Kranenburg, D. Molenaar, O. P. Kuipers, R. Leer, R. Tarchini, S. A. Peters, H. M. Sandbrink, M. W. E. J. Fiers, W. Stiekema, R. M. K. Lankhorst, P. A. Bron, S. M. Hoffer, M. N. N. Groot, R. Kerkhoven, M. de Vries, B. Ursing, W. M. de Vos, and R. J. Siezen. 2003. Complete genome sequence of Lactobacillus plantarum WCFS1. Proc. Natl. Acad. Sci. USA 100:1990-1995. https://doi.org/10.1073/pnas.0337704100.

Li, C., J. Song, L. Kwok, J. Wang, Y. Dong, H. Yu, Q. Hou, H. Zhang, and Y. Chen. 2017. Influence of Lactobacillus plantarum on yogurt fermentation properties and subsequent changes during postfermentation storage. J. Dairy Sci. 100:2512-2525. https://doi.org/ 10.3168/jds.2016-11864.

Ma, C., G. Cheng, Z. Liu, G. Gong, and Z. Chen. 2016. Determination of the essential nutrients required for milk fermentation by Lactobacillus plantarum. Lebensm. Wiss. Technol. 65:884-889. https:// doi.org/10.1016/j.lwt.2015.09.003.

Østergaard, O., S. Melchior, P. Roepstorff, and B. Svensson. 2002. Initial proteome analysis of mature barley seeds and malt. Proteomics 2:733-739. https://doi.org/10.1002/1615-9861(200206)2: $6<733::$ AID-PROT733>3.0.CO;2-E.

Quigley, L., O. O'Sullivan, C. Stanton, T. P. Beresford, R. P. Ross, G. F. Fitzgerald, and P. D. Cotter. 2013. The complex microbiota of raw milk. FEMS Microbiol. Rev. 37:664-698. https://doi.org/10 $.1111 / 1574-6976.12030$

Sadek, Z. I., M. A. Abdel Rahman, M. S. Azab, O. M. Darwesh, and M. S. Hassan. 2018. Microbiological evaluation of infant foods quality and molecular detection of Bacillus cereus toxins relating genes. Toxicol. Rep. 5:871-877. https://doi.org/10.1016/j.toxrep 2018.08.013

Saguir, F. M., I. E. Loto Campos, and M. C. Manca de Nadra. 2008. Utilization of amino acids and dipeptides by Lactobacillus plantarum from orange in nutritionally stressed conditions. J. Appl. Microbiol. 104:1597-1604. https://doi.org/10.1111/j.1365-2672 2007.03708.x.

Seddik, H. A., F. Bendali, F. Gancel, I. Fliss, G. Spano, and D. Drider. 2017. Lactobacillus plantarum and its probiotic and food poten- tialities. Probiotics Antimicrob. Proteins 9:111-122. https://doi .org/10.1007/s12602-017-9264-z.

Taheri Garavand, A., S. Rafiee, and A. Keyhani. 2011. Mathematical modeling of thin layer drying kinetics of tomato influence of air dryer conditions. International Transaction Journal of Engineering, Management, and Applied Sciences and Technologies 2:147160.

Tian, F., Y. Xiao, X. Li, Q. Zhai, G. Wang, Q. Zhang, H. Zhang, and W. Chen. 2015. Protective effects of Lactobacillus plantarum CCFM8246 against copper toxicity in mice. PLoS One 10:e0143318. https://doi.org/10.1371/journal.pone.0143318.

Tian, F., Q. Zhai, J. Zhao, X. Liu, G. Wang, H. Zhang, H. P. Zhang, and W. Chen. 2012. Lactobacillus plantarum CCFM8661 alleviates lead toxicity in mice. Biol. Trace Elem. Res. 150:264-271. https:/ /doi.org/10.1007/s12011-012-9462-1.

Yang, H., D. Hewes, S. Salaheen, C. Federman, and D. Biswas. 2014. Effects of blackberry juice on growth inhibition of foodborne pathogens and growth promotion of Lactobacillus. Food Control 37:15-20. https://doi.org/10.1016/j.foodcont.2013.08.042.

Yang, Q., X. Pan, W. Kong, H. Yang, Y. Su, L. Zhang, Y. Zhang, Y. Yang, L. Ding, and G. Liu. 2010. Antioxidant activities of malt extract from barley (Hordeum vulgare L.) toward various oxidative stress in vitro and in vivo. Food Chem. 118:84-89. https://doi.org/ 10.1016/j.foodchem.2009.04.094.

Yin, R., Q. Zhai, L. Yu, Y. Xiao, G. Wang, R. Yu, F. Tian, and W. Chen. 2016. The binding characters study of lead removal by Lactobacillus plantarum CCFM8661. Eur. Food Res. Technol. 242:1621-1629. https://doi.org/10.1007/s00217-016-2661-9.

Yu, L., Q. Zhai, F. Tian, X. Liu, G. Wang, J. Zhao, H. Zhang, A. Narbad, and W. Chen. 2016. Potential of Lactobacillus plantarum CCFM639 in protecting against aluminum toxicity mediated by intestinal barrier function and oxidative stress. Nutrients 8:783. https://doi.org/10.3390/nu8120783.

Zhai, Q., G. Wang, J. Zhao, X. Liu, A. Narbad, Y. Q. Chen, H. Zhang, F. Tian, and W. Chen. 2014. Protective effects of Lactobacillus plantarum CCFM8610 against chronic cadmium toxicity in mice indicate routes of protection besides intestinal sequestration. Appl. Environ. Microbiol. 80:4063-4071. https://doi.org/10.1128/AEM .00762-14.

Zhang, H., J. Liu, Y. Cao, and Y. Wang. 2013. Effects of particle size on lignite reverse flotation kinetics in the presence of sodium chloride. Powder Technol. 246:658-663. https://doi.org/10.1016/j .powtec.2013.06.033.

Zhang, J., P. Ma, X. Zhang, B. Wang, J. Wu, and X. Xing. 2018. Isothermal drying kinetics of paddy using thermogravimetric analysis. J. Therm. Anal. Calorim. 134:2359-2365. https://doi.org/10 .1007/s10973-018-7716-7.

\section{ORCIDS}

Feng Hang ๑ https://orcid.org/0000-0002-8771-9573 Yuanzhi Jiang ๑ https://orcid.org/0000-0001-9352-3545 Liwen Yan (1) https://orcid.org/0000-0002-6815-3930 Qing Hong ๑ https://orcid.org/0000-0002-7809-3476 Wenwei Lu $\odot$ https://orcid.org/0000-0002-8636-9815 Jianxin Zhao @ https://orcid.org/0000-0003-4516-0799 Hao Zhang ๑ https://orcid.org/0000-0002-9192-4684 Wei Chen ( https://orcid.org/0000-0003-3348-4710 